Research Article

\title{
An Industrial B-Mode Phased Array Ultrasonic Imaging Reconstruction Algorithm Based on FRI Sampling
}

\author{
Guangzhi Dai $\mathbb{D}^{1},{ }^{1}$ Zhiyong $\mathrm{He} \mathbb{D}^{\circ},{ }^{2}$ and Song $\operatorname{Lin}^{3}$ \\ ${ }^{1}$ School of Artificial Intelligence, Shenzhen Polytechnic, Shenzhen 518055, China \\ ${ }^{2}$ School of Mechanical and Electric Engineering, Soochow University, Suzhou 215021, China \\ ${ }^{3}$ School of Mechatronics Engineering, Harbin Institute of Technology, Harbin 150001, China \\ Correspondence should be addressed to Zhiyong He; he-zhiyong@139.com
}

Received 18 March 2020; Accepted 9 June 2021; Published 17 September 2021

Academic Editor: Rafael Morales

Copyright (c) 2021 Guangzhi Dai et al. This is an open access article distributed under the Creative Commons Attribution License, which permits unrestricted use, distribution, and reproduction in any medium, provided the original work is properly cited.

Firstly, a novel FRI sampling model has been proposed according to the characteristics of ultrasonic signals. The model has the advantages such as good stability, strong antinoise ability, simple circuit implementation, and fewer preconditions, compared to the traditional methods. Then, in order to verify the validity of the sampling model, the method is applied to B-type ultrasonic imaging, and a B-type phased array ultrasonic imaging algorithm based on FRI sampling model is proposed. Finally, the algorithm simulation experiment is designed, and the results show that the sampling point required by the proposed FRI sampling model is only $0.1 \%$ of the traditional B-type phased array ultrasonic imaging method, and the sampling frequency of the proposed ultrasonic imaging algorithm is only $0.0077 \%$ of the traditional B-type ultrasonic imaging method. Additionally, the experiment result indicates that this algorithm is more applicable to phased array ultrasonic imaging than the SOS filter is.

\section{Introduction}

With the development of ultrasonic testing technology, the number of transducers and the amount of recorded data have been increasing rapidly. Theories proposed in recent years, such as CS (compressed sensing), can break through the limitation of traditional sampling theories and obtain an accurate reconstruction of signals by sampling of sparse signals with lower frequencies. Such sampling method is called sub-Nyquist sampling or undersampling. An undersampling method develops fast and has made great progress in many practical applications: such as the single-pixel camera developed by Rice University, MRI RF pulse device and coded aperture camera developed by MIT, DNA microarray sensor developed by Illinois State University, and CS filter and chaotic device developed by Chinese Academy of Sciences. However, in the field of ultrasonic imaging, the applications of the undersampling have been rarely reported.

At present, researches on ultrasonic imaging technology based on the undersampling theory can be summarized into two categories:
(1) Researches focusing on the improvement of the speed and quality of imaging, where two methods are developed to improve the imaging resolution: one of them changes the direction of the transducer, such as sound velocity focusing method; the other is postprocessing data, such as filtering and deconvolution methods. Due to the limitation of manufacturing technology and procedures, it is difficult for the transducers to get smaller focuses, which usually produce huge scanning data and therefore affect the postprocessing of the data. In the postprocessing of the data, the superresolution technology becomes a feasible and promising method [1-3]. However, the high resolution image of classic superresolution algorithm is often constructed from the low-resolution image; due to the deficiency of inherent data acquisition conditions and the problem of noise accumulation, it is hard to implement the algorithm. Therefore, a new theory is required to break through the above difficulties, so that the above problems can be solved. The appearance of the CS theory has laid a theoretical 
foundation for rapid high-resolution ultrasonic imaging, attracting a lot of attentions from many researchers, such as Friboulet et al. [4-17] and Eldar et al. [18-34].

(2) The researches focusing on a reduction of the volume and energy-consumption of imaging device: these researches are mainly concentrated on two aspects, one is the undersampling imaging technology based on the CS theory, proposed by Friboulet et al.; the other is the undersampling imaging technology based on FRI sampling, proposed by Eldar et al.

A fast imaging method based on undersampling theory is a research hotspot in the field of ultrasound imaging in recent years. Friboulet et al. first introduced CS theory into the field of medical ultrasound imaging in 2010 [4]. Using wavelet transform basis function and wave atom basis function as the sparse representation basis of ultrasonic image, the sparse imaging of ultrasonic image is realized. Subsequently, Friboulet et al. conducted in-depth research on CS in the field of medical ultrasound imaging [5-10], mainly carried out sparse analysis of medical ultrasound images in $K$ space, and discussed in detail the reconstruction performance of different recovery algorithms based on CS for ultrasonic echo signal and extended its application to three-dimensional ultrasound imaging. In 2013, Friboulet et al. introduced the distributed CS theory $[11,12]$ into medical ultrasound imaging. They sparsely represent the echo signals of each transducer array on the same orthogonal basis of Fourier transform. Using the correlation between signals, joint sparse model and joint data recovery algorithm achieve CS imaging [13]. In addition, Eldar et al. first applied FRI theory to ultrasound in 2011 and proposed a sparse sampling structure for ultrasound imaging [18-34]. The research is based on the finite rate of innovation (FRI) [35] sampling signal model proposed by Vetterli et al. FRI uses Gaussian sampling kernel to sample, which overcomes the limitation of Shannon sampling width. However, this structure is unstable, and the signal-to-noise ratio is not ideal. Therefore, Tur et al. used FRI principle to construct measurement matrix and realized ultrasonic data imaging under single channel sampling framework [23]. Later, the research team conducted research on multichannel ultrasonic data sampling and frequency domain sparse sampling [24] and even explored the superresolution ultrasonic reconstruction algorithm based on CS [29]. Guangming et al. of Xidian University [36, 37] and Chinese Academy of Sciences [38] also developed various applications of CS in ultrasonic imaging. To sum up, FRI theory is still in the stage of theoretical research, and FRI sampling based on ultrasonic signal is still in the stage of preliminary exploration. Therefore, there are still some key problems to be studied in theory and application.

In this paper, an under sampling method for ultrasonic testing signal is proposed, which is a multichannel indirect FRI sampling method based on ultrasonic signal. In each channel, the original signal is multiplied by the carrier to generate a new analog signal, and then, the new signal is integrated and sampled to obtain a group of linear transfor-

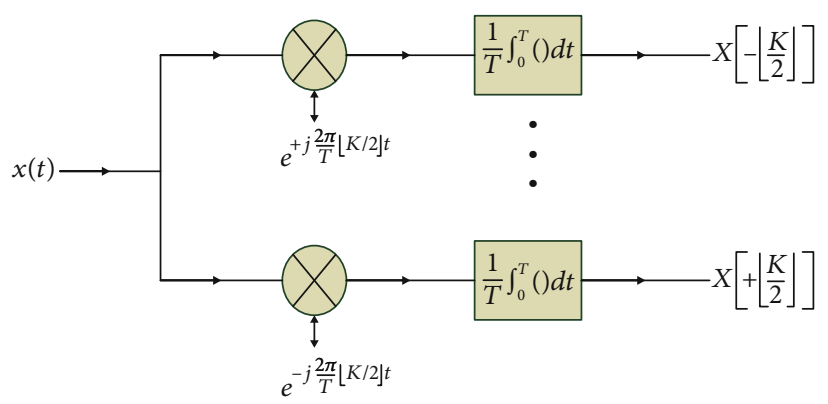

FIGURE 1: Multichannel direct sampling model.

mations about the Fourier coefficients of the original signal. Then, the Fourier coefficients of the original signal are obtained through linear transformation, and the signal is reconstructed by a spectral method. This scheme not only has the advantages of high stability and strong antinoise ability but also has the advantages of simple circuit structure, less need for prior knowledge, and much lower sampling frequency than traditional sampling methods. In order to verify the effectiveness of the proposed FRI sampling model, this method is applied to B-mode ultrasonic imaging, and a Bmode phased array ultrasonic imaging algorithm based on the FRI sampling model is proposed. The algorithm is based on the multichannel indirect FRI sampling model of ultrasonic signal and combined with the line scan mathematical model of B-mode ultrasonic imaging, so as to derive the Bmode ultrasonic imaging algorithm. Finally, the simulation experiment of B-mode ultrasonic imaging was designed according to this algorithm, and the results showed that the sampling points required by the proposed FRI sampling model was $0.1 \%$ of traditional B-mode phased array ultrasonic imaging and the sampling frequency of the proposed ultrasonic imaging algorithm was $0.0077 \%$ of that of the traditional B-mode ultrasonic imaging scheme.

\section{Basic Principle of Multichannel FRI Sampling}

2.1. Multichannel FRI Direct Sampling Model. According to the FRI theory, for FRI signals, as long as $K$ Fourier coefficients are known, the signals can be reconstructed according to the spectrum analysis. Therefore, getting the Fourier coefficient of FRI signals is the key of the problem.

The direct sampling model of Fourier coefficient is shown as Figure 1; $T$ is the sampling period, that is, the time length of limited FRI signals; $K$ is odd. For each channel, the original signal $x(t)$ is multiplied by a complex exponential function, which is the carrier signal; then its integral is divided by $T$ to get the Fourier coefficient vector $x$ of the channel. However, the model has an obvious disadvantage; that is, the frequency of the carrier function of each channel is different, which brings difficulties to circuit design.

2.2. Multichannel FRI Indirect Sampling Model. A Fourier coefficient indirect sampling model is shown as Figure 2, which is more practical than the direct sampling model. First, it has better robustness. The sampling result of each 


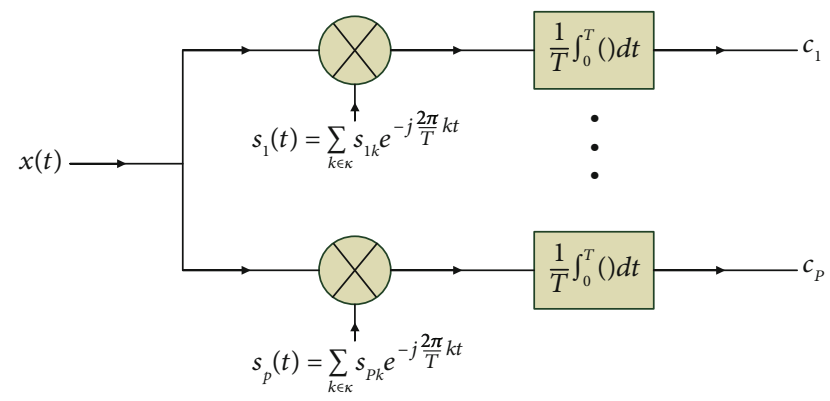

FIGURE 2: FRI indirect sampling model.

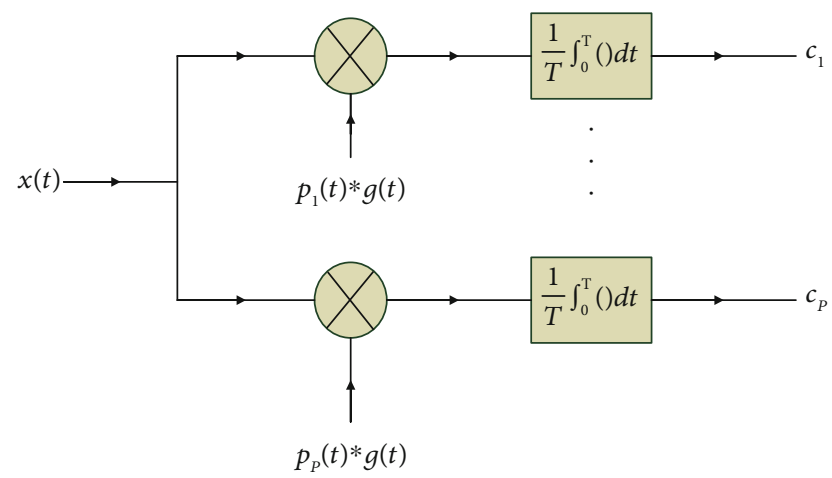

FIGURE 3: Multichannel FRI indirect sampling model of ultrasonic signal.

channel is the sum of a number of Fourier coefficients and will not affect the accuracy of the whole signal reconstruction due to the failure of one channel or more; second, it simplifies the carrier signal generation circuit, in which the carrier frequency components of each channel are the same, but the coefficients are different.

Its mathematical derivation is as follows:

Assume that there are $P$ channels, and according to Figure 2, the carrier signal of channel $i$ is

$$
s_{i}(t)=\sum_{k \in \mathcal{K}} s_{i k} e^{-j(2 \pi / T) k t},
$$

where the coefficient $s_{i}(t)=\sum_{k \in \kappa} s_{i k} e^{-j(2 \pi / T) k t}$ of each channel is different; assume that the sampling vector $c=\left\{c_{1}, c_{2}, \cdots\right.$, $\left.c_{p}\right\}$; then the sampling point of channel $i$ is

$$
c_{i}=\frac{1}{T} \int_{0}^{T} x(t) \sum_{k \in \mathcal{K}} s_{i k} e^{-j(2 \pi / T) k t} d t=\sum_{k \in \mathcal{K}} s_{i k} x[k] .
$$

Set $S$ as the matrix of $P \times K$, the element $(i, k)$ is $S_{i k}$, and then formula (2) can be written as the matrix form:

$$
c=S_{x}
$$

where $x$ is the Fourier coefficient vector and, if and only if column $S$ is full rank, that is, $P \geq K, S$ is left reversible; then,

$$
x=S^{+} c
$$

Hybrid matrix $S$ is determined by the selected carrier signal $S_{i}(t)$. Therefore, the Fourier coefficient direct sampling method is a special case of the Fourier coefficient indirect sampling method, where $P=K$ and $S=I$.

\section{Design of Multichannel FRI Indirect Sampling Model of Ultrasonic Signal}

Based on the practical application of FRI signal basic sampling model and ultrasonic signal, the designed sampling model is shown as Figure 3. In this model, the actual carrier signal $s_{i}(t)$ is generated from square wave signal $p_{i}(t)$ by means of filtering, and matrix $A$ generated by square wave signal and hybrid matrix $S$ generated by carrier signal are obtained by calculation.

3.1. Design of Square Signal $\boldsymbol{p}_{\boldsymbol{i}}(\boldsymbol{t})$. The mathematical model of square signal is shown as follows:

$$
p_{i}(t)=\sum_{m \in \mathbb{Z}} \sum_{n=0}^{N-1} a_{i}[n] p\left(\frac{t-n T}{N-m T}\right)
$$

where $a_{i}[n]$ is the coefficient vector whose length is $N$ and its element can only be ${ }_{-}^{+} 1$; set $P=N=K$; then $p(t)$ is

$$
p(t)= \begin{cases}1, & t \in\left[0, \frac{T}{N}\right], \\ 0, & t \notin\left[0, \frac{T}{N}\right] .\end{cases}
$$

Its continuous Fourier transformation is

$$
p(\omega)=\frac{T}{N} e^{-j(T / 2 N) \omega} \cdot \sin c\left(\frac{T}{2 \pi N} \omega\right) .
$$

3.2. Design of Carrier Signal $\boldsymbol{s}_{\boldsymbol{i}}(\boldsymbol{t})$. According to formula (5), $p_{i}(t)$ is a function with the period of $T$. Its Fourier efficient is shown as follows:

$$
p_{t}(t)=\sum_{k \in \mathbb{Z}} d_{i}[k] e^{-j(2 \pi / T) k},
$$

where the Fourier coefficient $d_{i}[k]$ is worked out according to the following formula:

$$
\mathrm{d}_{i}[k]=\frac{1}{T} \int_{0}^{T} p_{i}(t) e^{-j(2 \pi / T) k t} d t .
$$

It can be seen that the formula is in the form of infinite addition; therefore, to get $s_{i}(t)$, a filter $g(t)$ must be added to filter $p_{i}(t)$ :

$$
s_{i}(t)=p_{i}(t) * g(t) .
$$


However, to achieve this function, $g(t)$ must have the following restrictions:

$$
G(\omega)=\left\{\begin{array}{l}
\text { nonzero, } \quad \omega=\frac{2 \pi}{T} k, k \in \kappa, \\
0, \quad \omega \neq \frac{2 \pi}{T} k, k \notin \kappa \\
\text { arbitrary, } \quad \text { elsewhere, }
\end{array}\right.
$$

where $G(\omega)$ is the continuous Fourier transformation of the filter $g(t)$ and $g(t)$ was selected as the ideal low-pass filter; we can get

$$
\begin{aligned}
s_{i}(t) & =\sum_{k \in \mathbb{Z}} d_{i}[k] G\left(\frac{2 \pi}{T} k\right) e^{j(2 \pi / T) k t} \\
& =\sum_{k \in \mathcal{K}} d_{i}[k] G\left(\frac{2 \pi}{T} k\right) e^{j(2 \pi / T) k t} .
\end{aligned}
$$

It can be seen from formulas (1) and (12) that

$$
s_{i k}=d_{i}\left[-k-\left\lfloor\frac{K}{2}\right\rfloor\right] \cdot G\left(\frac{2 \pi}{T}\left(-\left(k-\left\lfloor\frac{K}{2}\right\rfloor\right)\right)\right) \text {. }
$$

3.3. Design of Hybrid Matrix S. According to formulas (5) and (9), we can get

$$
\begin{aligned}
d_{i}[k] & =\frac{1}{T} \sum_{m \in Z} \sum_{n=0}^{N} a_{i}[n] \int_{0}^{T} p\left(\frac{t-n T}{N-m T}\right) e^{-j(2 \pi / T) k t} d t \\
& =\frac{1}{T} \sum_{n=0}^{N} a_{i}[n] \sum_{m \in Z} \int_{-m T}^{-(m-1) T} p\left(\frac{t-n T}{N}\right) e^{-j(2 \pi / T) k t} d t \\
& =\frac{1}{T} \sum_{n=0}^{N} a_{i}[n] \sum_{m \in Z} \int_{-\infty}^{\infty} p\left(\frac{t-n T}{N}\right) e^{-j(2 \pi / T) k t} d t \\
& =\frac{1}{T} \sum_{n=0}^{N} a_{i}[n] P\left(\frac{2 \pi}{T} k\right) e^{-j(2 \pi / T) k n} .
\end{aligned}
$$

With formulas (13) and (14), we can get

$$
S_{i k}=\frac{1}{T} \sum_{n=0}^{N-1} a_{i}[n] P\left(\frac{2 \pi}{T} k^{\prime}\right) G\left(\frac{2 \pi}{T} k^{\prime}\right) e^{-j(2 \pi / N) k^{\prime} n}
$$

Then, formula (15) can be expressed with the hybrid matrix as

$$
S=A W \Phi
$$

where $A$ is the matrix of $P \times N$, the element $(i, n) A_{\text {in }}=a_{i}[n]$, and $W$ is the diagonal matrix $w_{n k} e^{-j(2 \pi / N) k^{\prime} n}$ of the matrix of $N \times K$, where the diagonal element is

$$
\Phi_{k k}=\frac{1}{T} P\left(\frac{2 \pi}{T} k^{\prime}\right) G\left(\frac{2 \pi}{T} k^{\prime}\right)
$$

Substitute formula (7) into formula (17), we can get

$$
\Phi_{k k}=\frac{1}{N} e^{-j(\pi / N) k^{\prime}} \sin c\left(\frac{k^{\prime}}{N}\right) G\left(\frac{2 \pi}{T} k^{\prime}\right) .
$$

When $g(t)$ is the ideal low-pass filter, formula (18) can be written as

$$
\Phi_{k k}=\frac{1}{N} e^{-j(\pi / N) k^{\prime}} \sin c\left(\frac{k^{\prime}}{N}\right)
$$

3.4. Design of Matrix $A$. Matrix $A$ is generated according to the square signal. In the actual circuit, there is only one square signal generator, and the square signal on each channel is gotten by the delay of the square signal produced by the signal generator. In fact, the square signal on channel $(i+1)$ delays $T / N$ (that is, a square wave) than the square signal $i$. As a rule, set $P=N=K=2 \times L \times$ oversampling +1 , where oversampling is the coefficient of oversampling. Then, matrix $A$ can be designed as follows: the elements in the first row are written (can only be set as +1 or -1 ) from the square wave signal of the first channel. Start with the second row; the vector in the $(i+1)$ row is cyclically shifted from the $i$ row vector to the left; for example, set $L=3$, oversampling $=1$, then $P=N=M=7$; if the square wave in the first channel is shown as Figure 4, then matrix $A$ can be written as formula (20), and the square signal and filtered carrier signal gotten by calculation are shown as Figure 5 .

$$
A_{7 \times 7}=\left(\begin{array}{ccccccc}
1 & -1 & 1 & -1 & -1 & 1 & 1 \\
-1 & 1 & -1 & -1 & 1 & 1 & 1 \\
1 & -1 & -1 & 1 & 1 & 1 & -1 \\
-1 & -1 & 1 & 1 & 1 & -1 & 1 \\
-1 & 1 & 1 & 1 & -1 & 1 & -1 \\
1 & 1 & 1 & -1 & 1 & -1 & -1 \\
1 & 1 & -1 & 1 & -1 & -1 & 1
\end{array}\right) .
$$

\section{Mathematical Model of B-Mode Phased Array Ultrasonic Imaging}

The B-mode ultrasonic imaging process is shown as Figure 6. Ultrasonic phased array probe is composed of several small wafers, which is called phased array element. During the transmission, the triggered signal is transmitted to the phased array controller, which will "command" the phased array unit to transmit an ultrasonic beam, which can be focused at different depths of detection. During the receiving process, the beam will be reflected back after encountering the obstacle; each phased array unit will process the received signal according to the calculated delay time and then adds up to get the reflected signal, which is 


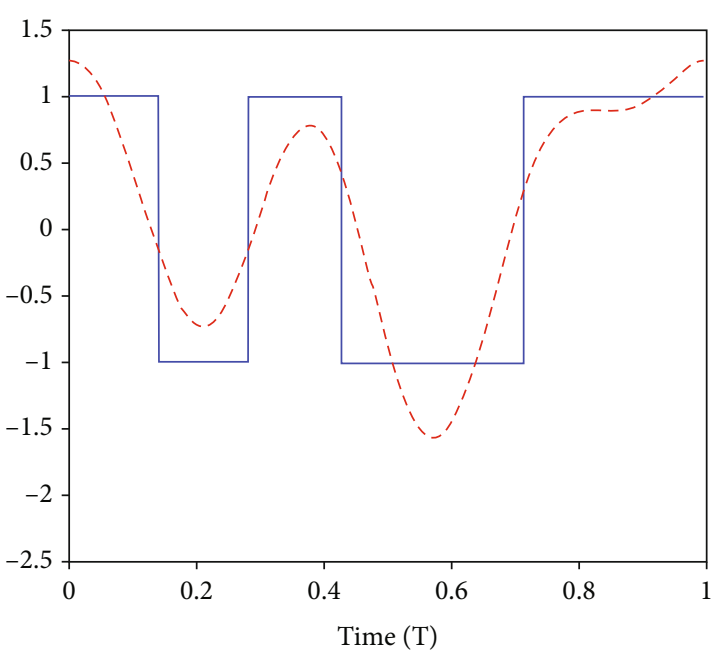

— Original modulating waveform

- - - Filtered modulating waveform

FIgURE 4: Square signal and filtered carrier signal.

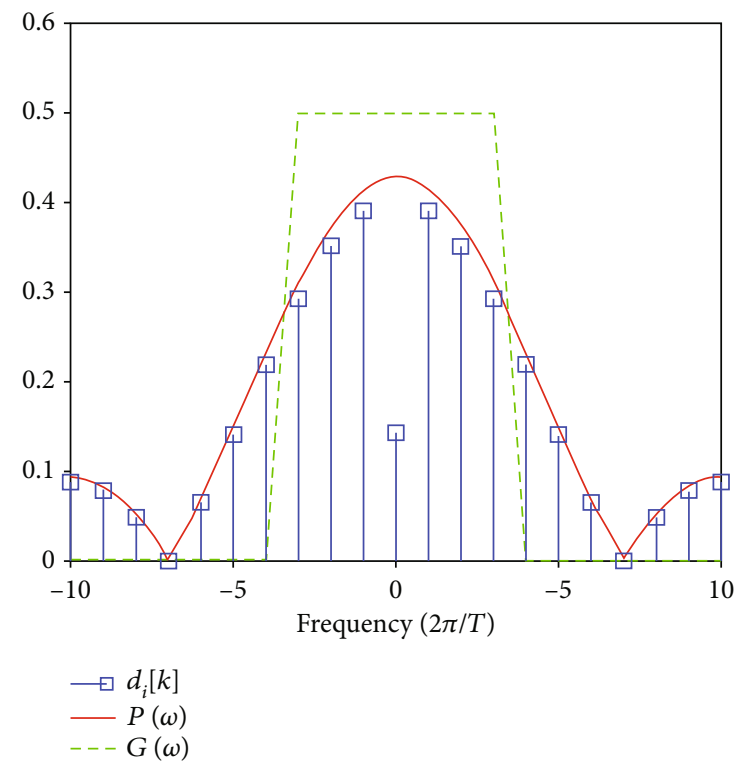

Figure 5: Square signal in the frequency domain and filtered carrier signal.

called a line scan. A two-dimensional image of the targeted area can be obtained for multiple line scans, which becomes B-mode ultrasonic imaging [39-41].

Figure 6 is the diagram of the line scan. $2 M+1$ phased array units distribute along the $x$-axis, the phased array unit $m_{0}$ was set as the origin, and $\delta_{m}$ expresses the distance from unit $m$ to unit $m_{0}$. The transmitted ultrasonic beam starts from the origin, and the included angle between the beam and $z$-axis is $\theta$; assume that the transmission time of the beam is 0 , and when time $t>0$, it arrives to the reflection point $(x, z)=(c t \sin \theta, c t \cos \theta)$, where $c$ is the ultrasonic velocity. When the beam gets the reflection point, it will be received by other phased array unit except for returning

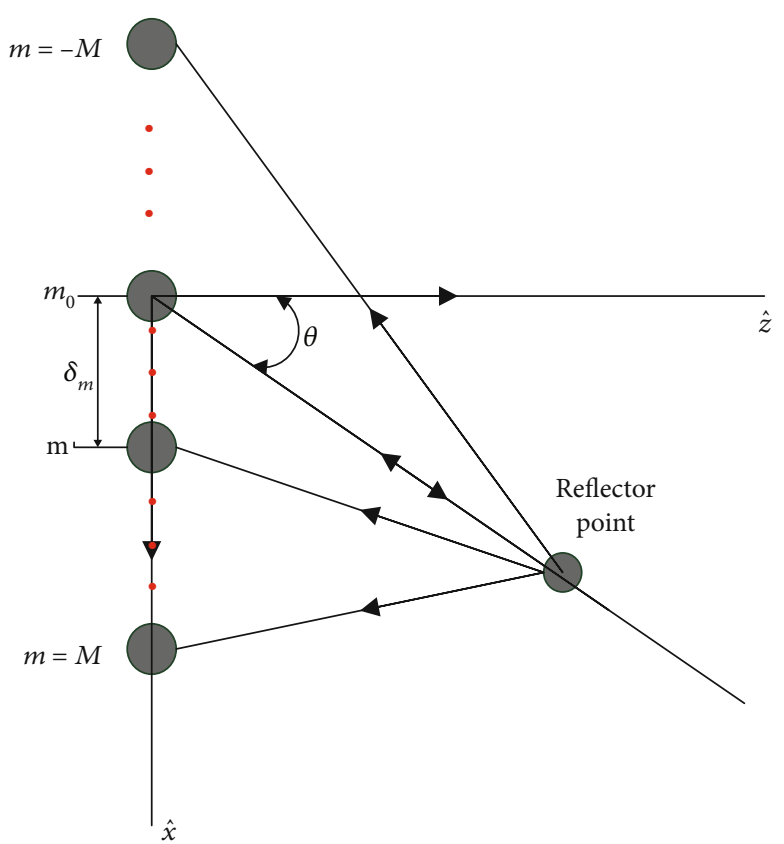

Figure 6: Line scan diagram.

units $m_{0}$ along the original path, where the distance of echo from the reflection point to the unit $m$ should be

$$
d_{m}(t ; \theta)=\sqrt{(c t \cos \theta)^{2}+\left(\delta_{m}-c t \sin \theta\right)^{2}} .
$$

Therefore, it can be calculated that the time of echo from the reflection point to unit $m$ is

$$
\widehat{\tau}_{m}(t, \theta)=t+\frac{d_{m}(t ; \theta)}{c}=t+\sqrt{t^{2}+4\left(\frac{\delta_{m}}{c}\right)^{2}-t \sin \theta} .
$$

According to formula (22), the actual lag time of the signals received by each unit is different so as to align the signals on the time axis. Delay the signals $\varphi_{m}(t)$ received by each phased array unit for a period of time to obtain the adjusted signals:

$$
\widehat{\varphi}_{m}(t ; \theta)=\varphi_{m}\left(\frac{\widehat{\tau}_{m}(t ; \theta)}{2}\right)
$$

Then, we can get the following after the calculation:

$$
\widehat{\varphi}_{m}(t ; \theta)=\varphi_{m}\left(\frac{1}{2}\left(t+\sqrt{t^{2}+4\left(\frac{\delta_{m}}{c}\right)^{2}-t \sin \theta}\right)\right)
$$




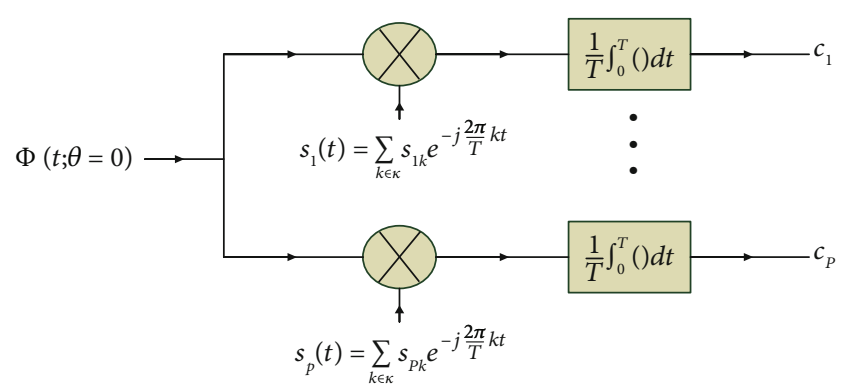

FIGURE 7: Ultrasonic signal multichannel FRI indirect sampling model.

When $\theta=0$, the line scanning is called parallel scanning, and the scanning line is parallel to the axis. At this time, formula (24) can be written as

$$
\widehat{\varphi}_{m}(t ; \theta=0)=\varphi_{m}\left(\frac{1}{2}\left(t+\sqrt{t^{2}+4\left(\frac{\delta_{m}}{c}\right)^{2}}\right)\right)
$$

At this time, $\delta_{m}$ represents the distance between unit $m$ to the scanning line. Add all phased array unit $\widehat{\varphi}_{m}(t ; \theta)$; then, we get the mathematical model of the line scanning:

$$
\begin{aligned}
\Phi(t ; \theta)= & \sum_{m=-M}^{M} \widehat{\varphi}_{m}(t ; \theta)=\sum_{m=-M}^{M} \varphi_{m} \\
& \cdot\left(\frac{1}{2}\left(t+\sqrt{t^{2}+4\left(\frac{\delta_{m}}{c}\right)^{2}-t \sin \theta}\right)\right),
\end{aligned}
$$

where the mathematical model of the parallel scanning is

$$
\Phi(t ; \theta=0)=\sum_{m=-M}^{M} \varphi_{m}\left(\frac{1}{2}\left(t+\sqrt{t^{2}+4\left(\frac{\delta_{m}}{c}\right)^{2}}\right)\right) .
$$

\section{Ultrasonic Imaging Algorithm Based on FRI Indirect Sampling}

In the case of considering only parallel line scanning, since $\Phi(t ; \theta=0)$ is also a FRI signal, it is written as follows:

$$
\Phi(t ; \theta=0)=\sum_{l=1}^{L} b_{l} h\left(t-t_{l}\right)
$$

Indirect sampling of the Fourier coefficients is as shown in Figure 7.
We can get the sampling point of the channel $q$ :

$$
\begin{aligned}
c_{q}= & \frac{1}{T} \int_{0}^{T}\left\{\sum_{k \in \mathcal{K}}\left(s_{q, k} e^{-j(2 \pi / T) k t}\right)\right\} \\
& \cdot\left\{\sum_{m=-M}^{M} \varphi_{m}\left(\frac{1}{2}\left(t+\sqrt{t^{2}+4\left(\frac{\delta_{m}}{c}\right)^{2}}\right)\right)\right\} d t .
\end{aligned}
$$

After deformation, formula (29) can be written as

$$
c_{q}=\sum_{m=-M}^{M}\left\{\frac{1}{T} \int_{0}^{T} \widehat{s}_{q, m}(t) \varphi_{m}(t) d t\right\}
$$

where $\widehat{s}_{q, m}(t)$ is defined as follows:

$$
\begin{aligned}
\widehat{s}_{q, m}(t) \triangleq & {\left[1+\left(\frac{\delta_{m}}{c t}\right)^{2}\right]\left\{\sum_{k \in \mathcal{K}} s_{q, k} e^{-j(2 \pi / T) k\left(t-(1 / t)\left(\delta_{m} / c\right)^{2}\right)}\right\} } \\
& \cdot u\left(t-\left|\frac{\delta_{m}}{c}\right|\right), \quad \text { for all } 1 \leq q \leq p,-M \leq m \leq M,
\end{aligned}
$$

where $\overparen{T}$ is defined as follows:

$$
\widehat{T}=\max _{m \in\{-M, \cdots, M\}}\left\{\frac{1}{2}\left(t+\sqrt{t^{2}+4\left(\frac{\delta_{m}}{c}\right)^{2}}\right)\right\}
$$

where $u(t)$ is the step function paraded and it is defined as follows:

$$
u(t)= \begin{cases}1, & t \geq 0 \\ 0, & \text { else }\end{cases}
$$

Set

$$
\overparen{t}=\frac{1}{2}\left(t+\sqrt{t^{2}+4\left(\frac{\delta_{m}}{c}\right)^{2}}\right) .
$$

Then,

$$
t=\overparen{t}-\frac{1}{\overparen{t}}\left(\frac{\delta_{m}}{c}\right)^{2}
$$




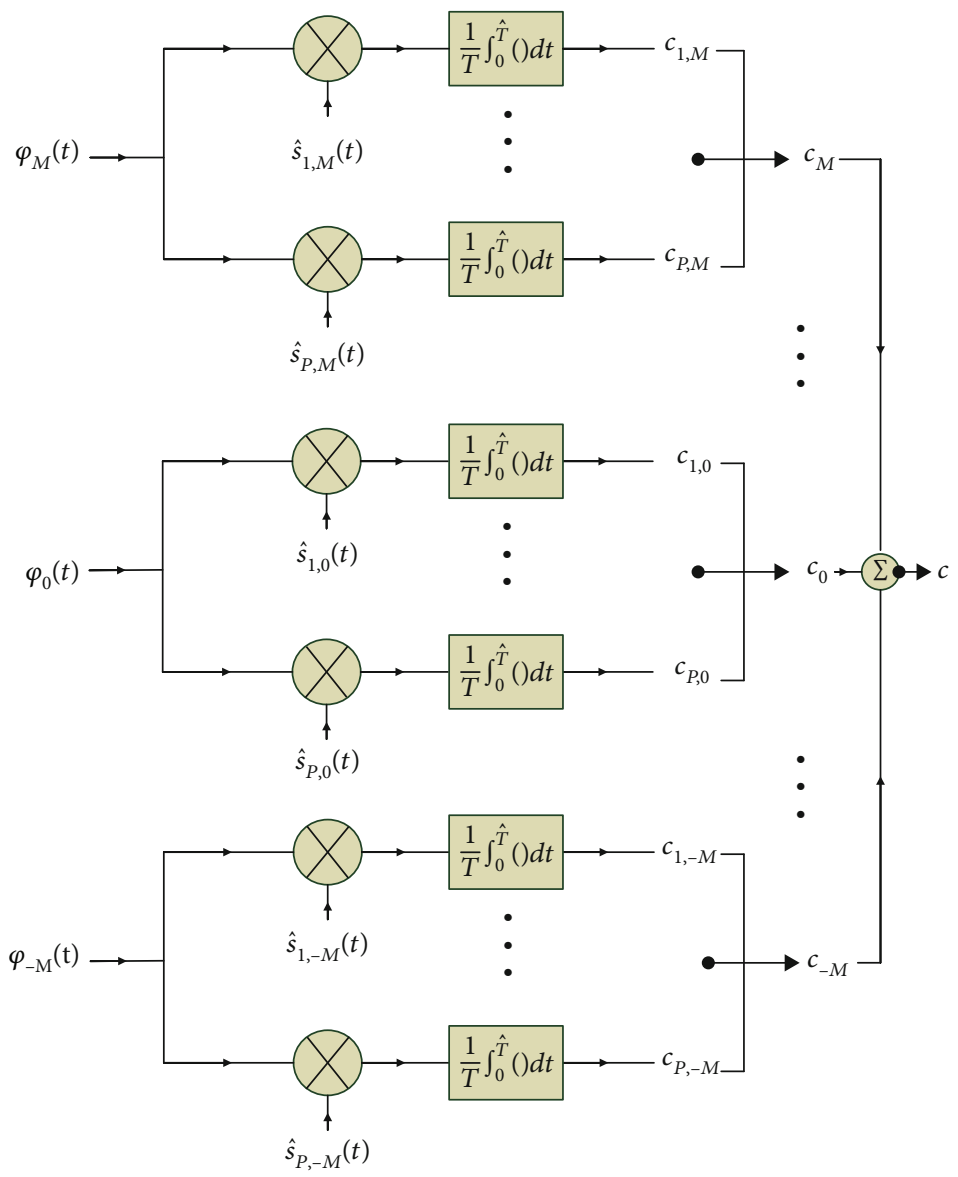

FIgURE 8: B-mode ultrasonic imaging FRI sampling model.

Substitute formula (35) into formula (29), then we can get

$$
\begin{aligned}
c_{q}= & \frac{1}{T} \int_{0}^{\widehat{T}}\left\{\sum_{k \in \kappa}\left(s_{q, k} e^{-j(2 \pi / T) k\left(\widehat{t}-(1 / \widehat{t})\left(\delta_{m} / c\right)^{2}\right)}\right)\right\} \\
& \cdot\left\{\sum_{m=-M}^{M} \varphi_{m}(\widehat{t})\right\} d\left(\widehat{t}-\frac{1}{\widehat{t}}\left(\frac{\delta_{m}}{c}\right)\right)^{2} \\
= & \sum_{m=-M}^{M}\left\{\frac{1}{T} \int_{0}^{T}\left[1+\left(\frac{\delta_{m}}{c \widehat{t}}\right)^{2}\right]\right. \\
& \cdot\left\{\sum_{k \in \kappa} s_{q, k} e^{-j(2 \pi / T) k\left(\widehat{t}-(1 / \widehat{t})\left(\delta_{m} / c\right)^{2}\right)}\right\} \\
& \left.\cdot u\left(\widehat{t}-\left|\frac{\delta_{m}}{c}\right|\right) \varphi_{m}(\widehat{t}) d \widehat{t}\right\} .
\end{aligned}
$$

Set $\overparen{t}=t$; then write formula (36) as formula (30).

In conclusion, the mathematical model of B-mode ultrasonic imaging FRI sampling is shown as Figure 8.

The specific algorithm to realize the model is as follows:

(1) Indirect sampling of the Fourier coefficients of the signals was received by each phased array unit, and we can get $c_{q, m}=\int_{0}^{T} \widehat{s}_{q, m}(t) \varphi_{m}(t) d t$; define the sampling vector of length $P$ asc $c_{m}=\left\{c_{1, m}, \cdots, c_{p, m}\right\}$

(2) After getting sampling vector of each phased array unit, add them; then, we get the final sampling vector $c=\sum_{m=-M}^{M} c_{m}$

(3) Calculate Fourier coefficient vector $x=s^{+} c$, where matrix $s$ is $p \times k$ and its $(i, k)$ element is $s_{q k}$

(4) Use formula $y=H^{-1} x$; we can get the equation set $y_{k}=\sum_{l=1}^{L} a_{l} e^{-j 2 \pi k t_{l} / t}, k \in \kappa$

(5) Use Matrix Pencil to solve the equation set; we can get $\left\{t_{l}\right\}_{l=1}^{L}$, and the reason for using Matrix Pencil rather than Annihilating Filter to solve the equation set is that Matrix Pencil can estimate the number $L$ of pulses

(6) Substitute $\left\{t_{l}\right\}_{l=1}^{L}$ into the equation set; use the least square method to solve $\left\{b_{l}\right\}_{l=1}^{L}$

(7) Use formula $\Phi(t ; \theta=0)=\sum_{l=1}^{L} b_{l} h\left(t-t_{l}\right)$ to reconstruct signal $\Phi(t ; \theta=0)$

\section{Simulation}

The emulation steps of the algorithm are as follows: 


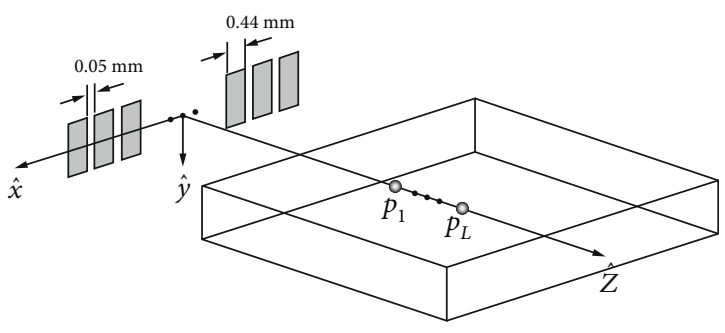

FIGURE 9: Working platform of ultrasonic imaging.

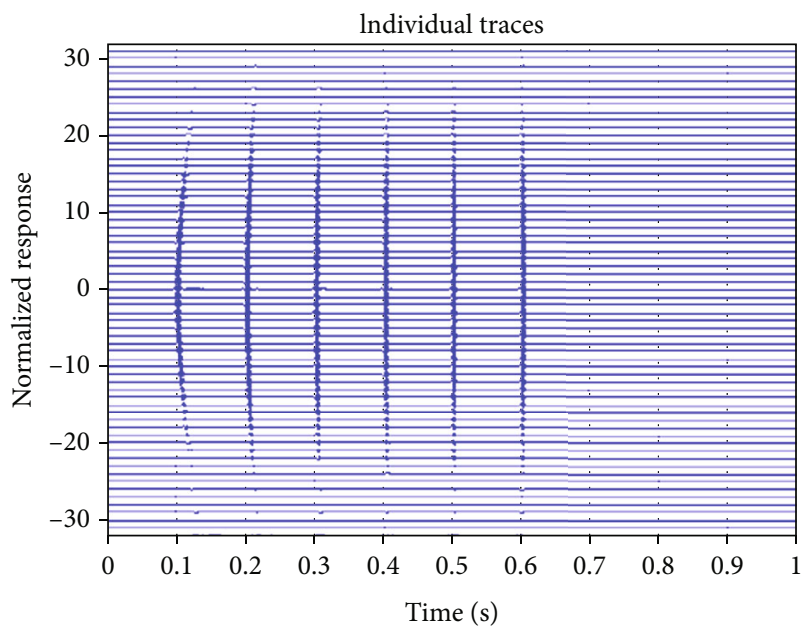

Figure 10: When $x=0$, the original signals are on the 65 line arrays.

(1) Use the software Field II to simulate the original signal of B-mode ultrasonic imaging, and the working platform is shown in Figure 9

The structure of the working platform is as follows. The platform consists of 65 line array units, marked as $\left\{m_{-32}\right.$, $\left.\cdots, m_{0}, \cdots, m_{32}\right\}$, the central frequency of the line array is $f_{0}$ $=3.5 \mathrm{MHz}$, the line array is arranged along axis $\widehat{x}$, the width of the line array is $c / f_{0}=0.44 \mathrm{~mm}$, and the height of it is $5 \mathrm{~mm}$, measured by the axis $\widehat{y}$; the spacing of the line array is $0.05 \mathrm{~mm}$, and the transmitting signal of the line array is the sine function of two periods, with a frequency of $f_{0}$, that is $\sin \left(2 \pi f_{0} t\right), 0 \leq t \leq 2 / f_{0}$; the focusing depth of the working platform is $r=70 \mathrm{~mm}$, and the sampling frequency is $f_{\mathrm{s}}=$ $100 \mathrm{MHz}$.

(2) The measured object is a cuboid $\{(x, y, z):|x| \leq 20$ $\mathrm{mm},|y| \leq 5 \mathrm{~mm}, 0 \leq z \leq 90 \mathrm{~mm}\}$ including 6 reflection points evenly spaced along the axis $\widehat{z}:(0,0,10)$, $(0,0,20),(0,0,30),(0,0,40),(0,0,50)$; and $(0,0,60)$

(3) This emulation is parallel line scanning, that is $\theta=0$, which is parallel to axis $\widehat{z}$ and is in plane $\widehat{z} o \widehat{x}$. Scan from $x=-20$ equally spaced to $x=20$, with a total of 50 scans, and we get $50 \times 65$ original signals. What Figure 10 shows is the 65 original signals by line scanning (signals on the 65 line scanning unit) when $x=0$

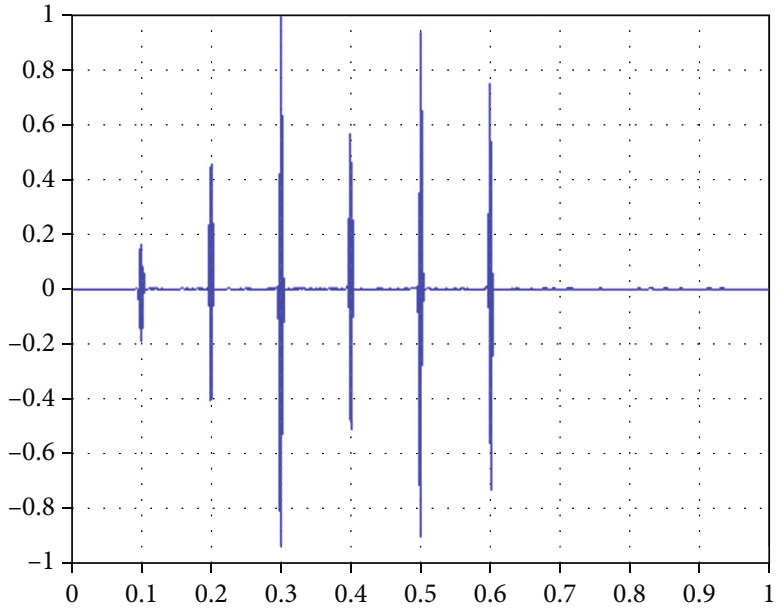

FiguRE 11: When $x=0$, the original signals are on the line array unit.

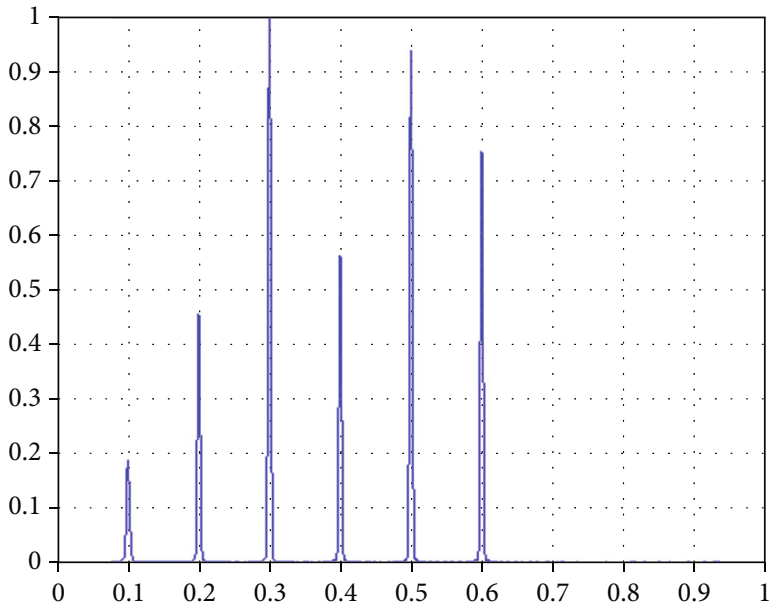

FIgURE 12: When $x=0$, the modulated $\operatorname{signal} \varphi_{m_{0}}(t)$ is on the line array unit.

(4) When $x=0$, the B-mode ultrasonic imaging process is as follows: as mentioned above, 65 signals are obtained, where the original signals of the line array units are shown as Figure 11

Since the original signal $h(t)$ is the sinusoidal pulse and its continuous Fourier transformation $H(w)$ are only two pulses. To ensure the reversibility of matrix $H$, the original signal needs to be modulated to make it become Gaussian pulse signal. The modulated signal $\varphi_{m_{0}}(t)$ is shown as Figure 12.

The modulated signal is gotten after modulating units on the 65 line array units; sampling vector $c$ is obtained by using the sampling model shown in Figure 7, and $\left\{t_{l}\right\}_{l=1}^{L}$ is obtained after calculation. In ultrasonic imaging, getting the delay vector means getting the location information of the reflecting point. As shown in Figure 13, when the delayed vector $\left.\Phi(t ; \theta=0)\right|_{x=0}$ is obtained, set the amplitude of all delayed vectors as 1 . 


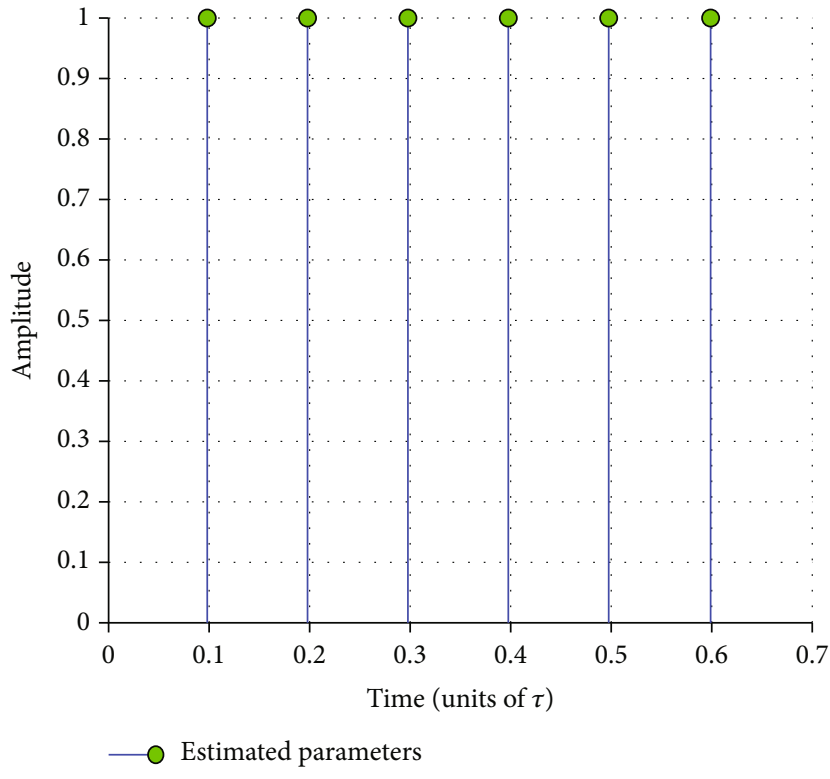

Figure 13: Delayed vector.

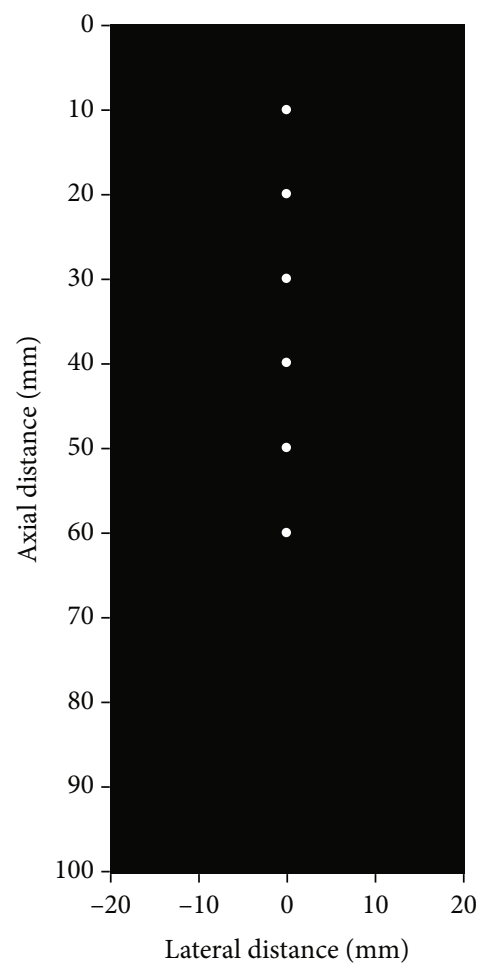

FIgURE 14: Reconstructed image.

(5) Thus, repeat step (4) for 50 scanning lines; we get 50 groups of delayed vectors of $\left.\Phi(t ; \theta=0)\right|_{x}, x=-20$, $-19.2, \cdots, 0, \cdots, 19.2,20$

(6) Use 50 groups of delayed vectors $\left.\left\{t_{l}\right\}_{l=1}^{6}\right|_{x}$, and the reconstructed image is shown as Figure 14

According to the above analysis process, the number of sampling points required by traditional B-mode ultrasonic imaging methods is $N_{\text {classic }}=\left(2 R_{\max } / c\right) \times f_{s} \times 50=(2 \times 0.1 /$ $1540) \times\left(100 \times 10^{6}\right) \times 50=649350$, while the number of sampling points by using this method is $N_{\text {sub-Nyquist }}=(2 \mathrm{~L}$ $+1) \times 50=(2 \times 6+1) \times 50=650$.

Therefore, the sampling points based on Fourier coefficient indirect sampling model are $650 / 649350=0.1 \%$ of that of traditional B-mode phased array ultrasonic imaging method, while the sampling frequency is $0.1 / 13=0.0077 \%$.

\section{Conclusion}

(1) A new FRI sampling model has been proposed based on ultrasonic signal. This model has the advantages such as good stability, simple circuit, and implementation

(2) A B-mode phased array ultrasonic imaging algorithm has been proposed based on the FRI sampling model. This algorithm is proposed on the basis of both the FRI sampling model and the mathematical model characteristics of B-mode phased array ultrasonic imaging

(3) The simulation program is designed for the algorithm, and the results show that the sampling point required by the proposed FRI sampling model is $0.1 \%$ of the traditional mode of B-mode phased array ultrasonic imaging, and the sampling frequency of the proposed ultrasonic imaging algorithm is $0.0077 \%$ of that of the traditional B-mode ultrasonic imaging

\section{Data Availability}

The raw/processed data required to reproduce these findings cannot be shared at this time as the data also forms part of an ongoing study.

\section{Conflicts of Interest}

The authors declare that they have no conflicts of interest.

\section{Acknowledgments}

This work was supported in part by the Natural Science Foundation of China under Grant 61671309, in part by the Guangdong Natural Science Foundation under Grant S2011010004487, and in part by the China Postdoctoral Science Foundation under Grant 2012M511551. The authors would like to thank Weiyi Lin, Chaoyi Dong, and Hongwei Sun for their contribution to this work.

\section{References}

[1] S. Mallat and Guoshen Yu, "Super-resolution with sparse mixing estimators," IEEE Transactions on Image Processing, vol. 19, no. 11, pp. 2889-2900, 2010.

[2] J. Yang, J. Wright, T. Huang, and Y. Ma, "Image superresolution as sparse representation of raw image patches," in IEEE conference on computer vision and pattern recognition (CVPR), pp. 1-8, Washington, DC USA, 2008. 
[3] Jianchao Yang, J. Wright, T. Huang, and Yi Ma, "Image superresolution via sparse representation," IEEE Transactions on Image Processing, vol. 19, no. 11, pp. 2861-2873, 2010.

[4] D. Friboulet, H. Liebgott, and R. Prost, "Compressive sensing for raw RF signals reconstruction in ultrasound," in 2010 IEEE International Ultrasonics Symposium, pp. 367-370, San Diego, CA, USA, 2002.

[5] C. Quinsac, A. Basarab, and D. Kouamé, "Frequency domain compressive sampling for ultrasound imaging," Advances in Acoustics and Vibration, vol. 2012, Article ID 231317, 16 pages, 2012.

[6] H. Liebgott, R. Prost, and D. Friboulet, "Pre-beamformed RF signal reconstruction in medical ultrasound using compressive sensing," Ultrasonics, vol. 53, no. 2, pp. 525-533, 2013.

[7] N. Dobigeon, A. Basarab, D. Kouamé, and J.-Y. Tourneret, "Regularized Bayesian compressed sensing in ultrasound imaging," in Proc. European Signal ProcessConf. (EUSIPCO), Bucharest, Romania, 2012.

[8] C. Quinsac, F. de Vieilleville, A. Basarab, and D. Kouamé, "Compressed sensing of ultrasound single-orthant analytical signals," in Proceedings of the IEEE International Ultrasonics Symposium (IUS'10), Orlando, FL, USA, 2010.

[9] C. Quinsac, A. Basarab, D. Kouamé, and J.-M. Grégoire, “3D compressed sensing ultrasound imaging," in Ultrasonics Symposium (IUS), pp. 363-366, San Diego, CA, USA, 2010.

[10] C. Quinsac, A. Basarab, J.-M. Girault, and D. Kouamé, “Compressed sensing of ultrasound images: sampling of spatial and frequency domains," in Signal Processing Systems (SIPS), pp. 231-236, San Francisco, CA, USA, 2010.

[11] D. Baron, M. B. Wakin, M. F. Duarte, S. Sarvotham, and R. G. Baraniuk, Distributed Compressed Sensing, Thermo Fisher, 2005.

[12] D. Baron, M. F. Duarte, S. Sarvotham, M. B. Wakin, and R. G. Baraniuk, "An information-theoretic approach to distributed compressed sensing," in Proc. 45rd Conference on Communication, Control, and Computing, proc.allerton, 2005.

[13] A. Basarab, H. Liebgott, O. Bernard, D. Friboulet, and D. Kouamé, "Medical ultrasound image reconstruction using distributed compressive sampling," in Biomedical Imaging (ISBI), pp. 1945-7928, San Francisco, CA, USA, 2013.

[14] H. Liebgott, A. Basarab, D. Kouame, O. Bernard, and D. Friboulet, "Compressive sensing in medical ultrasound," in Ultrasonics symposium (IUS), pp. 1-6, Dresden, Germany, 2012.

[15] A. Achim, B. Buxton, G. Tzagkarakis, and P. Tsakalides, "Compressive sensing for ultrasound RF echoes using astable distributions," in Engineering in Medicine and Biology Society (EMBC), pp. 4304-4307, Buenos Aires, Argentina, 2010.

[16] S. Hua, M. Yuchi, and M. Ding, "Compressed sensing for RF signal reconstruction in B-model ultrasound imaging," in Intelligent Computation and Bio-Medical Instrumentation (ICBMI), pp. 19-22, Wuhan, China, 2011.

[17] C. Quinsac, N. Dobigeon, A. Basarab, D. Kouamé, and J.Y. Tourneret, "Bayesian compressed sensing in ultrasound imaging," in Computational Advances in Multi-Sensor Adaptive Processing (CAMSAP), pp. 101-104, San Juan, PR, USA, 2011.

[18] N. Wagner, Y. C. Eldar, A. Feuer, G. Danin, and F. Z. Xampling, "Xampling in ultrasound imaging," in SPIE Medical Imaging, proc.allerton, 2011.
[19] M. Mishali and Y. C. Eldar, "From theory to practice: subNyquist sampling of sparse wideband analog signals," IEEE Journal of Selected Topics on Signal Processing, vol. 4, no. 2, pp. 375-391, 2010.

[20] M. Mishali, Y. C. Eldar, and A. Elron, "Xampling: signal acquisition and processing in union of subspaces," IEEE Transactions on Signal Processing, vol. 59, no. 10, pp. 4719-4734, 2011.

[21] T. Chernyakova and Y. C. Eldar, "Exploiting FRI signal structure for sub-Nyquist sampling and processing in medical ultrasound," in Acoustics, Speech and Signal Processing (ICASSP), South Brisbane, QLD, Australia, 2015.

[22] T. Michaeli and Y. C. Eldar, "Xampling at the rate of innovation," IEEE Transactions on Signal Processing, vol. 60, no. 3, pp. 1121-1133, 2012.

[23] R. Tur, Y. Eldar, and Z. Friedman, "Innovation rate sampling of pulse streams with application to ultrasound imaging," IEEE Transactions on Signal Processing, vol. 59, no. 4, pp. 18271842, 2011.

[24] R. T. Gedalyahu and Y. C. Eldar, "Multichannel sampling of pulse streams at the rate of innovation," IEEE Transactions on Signal Processing, vol. 59, no. 4, pp. 1491-1504, 2011.

[25] T. Chernyakova, Y. C. Eldar, and R. Amit, "Fourier domain beamforming for medical ultrasound," in Acoustics, Speech and Signal Processing (ICASSP), pp. 1520-6149, Vancouver, BC, Canada, 2013.

[26] A. Burshtein, M. Birk, T. Chernyakova, A. Eilam, A. Kempinski, and Y. C. Eldar, "Sub-Nyquist sampling and Fourier domain beamforming in volumetric ultrasound imaging," IEEE Transactions on Ultrasonics, Ferroelectrics, and Frequency Control, vol. 63, no. 5, pp. 703-716, 2016.

[27] T. Chernyakova and Y. C. Eldar, "Fourier-domain beamforming: the path to compressed ultrasound imaging," IEEE Transactions on Ultrasonics, Ferroelectrics, and Frequency Control, vol. 61, no. 8, pp. 1252-1267, 2014.

[28] Y. C. Eldar and G. Kutyniok, Compressed Sensing: Theory and Applications, Cambridge University Press, 2012.

[29] C. Bar-Zion, O. Tremblay-Darveau, D. A. Solomon, and Y. C. Eldar, "Super-resolution ultrasound imaging of vascular structures with high temporal resolution," IEEE Transactions on Medical Imaging, 2016.

[30] O. Solomon, R. Cohen, Y. Zhang et al., "Deep unfolded robust PCA with application to clutter suppression in ultrasound," IEEE Transactions on Medical Imaging, vol. 39, no. 4, pp. 1051-1063, 2018.

[31] R. Cohen and Y. C. Eldar, "Sparse convolutional beamforming for ultrasound imaging," IEEE Transactions on Ultrasonics, Ferroelectrics, and Frequency Control, vol. 65, no. 12, pp. 2390-2406, 2018.

[32] C. Bar-Zion, O. Tremblay-Darveau, D. A. Solomon, and Y. C. Eldar, "Fast vascular ultrasound imaging with enhanced spatial resolution and background rejection," IEEE Transactions on Medical Imaging, vol. 36, no. 1, pp. 169-180, 2017.

[33] M. Burshtein, T. Birk, A. Chernyakova, A. K. Eilam, and Y. C. Eldar, "Sub-Nyquist sampling and Fourier domain beamforming in volumetric ultrasound imaging," IEEE Transactions on Ultrasonics, Ferroelectrics, and Frequency Control, vol. 63, no. 5, pp. 703-716, 2016.

[34] M. Birk, A. Burshtein, T. Chernyakova et al., "Compressed 3D ultrasound imaging with 2D arrays," in 2014 IEEE International Conference on Acoustics, Speech and Signal Processing (ICASSP), pp. 6919-6923, Florence, Italy, May 2014. 
[35] M. Vetterli, P. Marziliano, and T. Blu, "Sampling signals with finite rate of innovation," IEEE Transactions on Signal Processing, vol. 50, no. 6, pp. 1417-1428, 2002.

[36] S. Guangming, C. Chongyu, J. Lin, X. Xie, and X. Chen, "Narrowband ultrasonic detection with high range resolution: separating echoes via compressed sensing and singular value decomposition," IEEE Transactions on Ultrasonics, Ferroelectrics, and Frequency Control, vol. 59, no. 10, pp. 2237-2253, 2012.

[37] S. Guangming, C. Xuyang, S. Xiaoxia, Q. Fei, and A. Ding, "Signal matching wavelet for ultrasonic flaw detection in high background noise," IEEE Transactions on Ultrasonics, Ferroelectrics and Frequency Control, vol. 58, pp. 776-787, 2011.

[38] L. Yi, Study on Ultrasonic Imaging Technology of Sparse Medicine, Institute of Acoustics, Chinese Academy of Sciences, Beijing, 2013.

[39] T. L. Szabo, Diagnostics Ultrasound Imaging: Inside Out, Elsevier Academic Press, Burlington, 2004.

[40] J. A. Jensen, "Ultrasound imaging and its modeling," Topics in Applied Physics, vol. 84, no. 1, pp. 135-165, 2002.

[41] J. A. Jensen, Linear Description of Ultrasound Imaging Systems, Technical University of Denmark: the International Summer School on Advanced Ultrasound Imaging, 1999. 